\title{
The 1997 Kagoshima (Japan) earthquake doublet: A quantitative analysis of aftershock rate changes
}

\author{
J. Woessner, ${ }^{1}$ E. Hauksson, ${ }^{2}$ S. Wiemer, ${ }^{1}$ and S. Neukomm ${ }^{1}$ \\ Received 17 October 2003; revised 22 December 2003; accepted 30 December 2003; published 10 February 2004.
}

[1] We quantitatively map relative rate changes for the aftershock sequence following the second mainshock of the 1997 earthquake doublet $\left(\mathrm{M}_{\mathrm{W}}=6.1, \mathrm{M}_{\mathrm{W}}=6.0\right)$ in the Kagoshima province (Japan). Using the spatial distribution of the modified Omori law parameters for aftershocks that occurred during the 47.8 days between the two mainshocks, we forecast the aftershock activity in the next 50 days and compare it to the actually observed rates. The relative rate change map reveals four regions with statistically significant relative rate changes - three negative and one positive. "Our analysis suggests that the coseismic rate changes for off-fault aftershocks could be explained by changes in static stress. However, to explain the activation and deactivation of on-fault seismicity, other mechanism such as unusual crustal properties and the presence of abundant crustal fluids are required." INDEX TERMS: 7215 Seismology: Earthquake parameters; 7223 Seismology: Seismic hazard assessment and prediction; 7230 Seismology: Seismicity and seismotectonics. Citation: Woessner, J., E. Hauksson, S. Wiemer, and S. Neukomm (2004), The 1997 Kagoshima (Japan) earthquake doublet: A quantitative analysis of aftershock rate changes, Geophys. Res. Lett., 31, L03605, doi:10.1029/2003GL018858.

\section{Introduction}

[2] Understanding how earthquakes are affected by sudden changes in the state of stress in their immediate vicinity provides insight into the relationship between static stress changes and earthquake occurrence. If aftershocks are in part caused by stress changes from their mainshock, changes in their rate may reflect alterations in the state of stress in their immediate vicinity. Recently, Stein [2003] provided a review of how adjacent earthquakes interact through static stress changes, invoking a combination of rate and state friction [Dieterich, 1994] and Coulomb Stress changes [King et al., 1994]. In particular, Stein [2003] and Toda and Stein [2003] suggested that the second mainshock of the 1997 Kagoshima earthquake sequence in Japan decreased the rate of off-fault aftershocks from the first mainshock due to a stress drop caused by the second main event.

[3] The objective of our study is to confirm spatial changes in the rate of aftershocks following the second mainshock and to investigate whether or not these changes are statistically significant. Different from previous studies, we determine the spatial distribution of relative rate changes

\footnotetext{
${ }^{1}$ Institute of Geophysics, ETH Zurich, Switzerland.

${ }^{2}$ California Institute of Technology, Los Angeles, California, USA.
}

Copyright 2004 by the American Geophysical Union. 0094-8276/04/2003GL018858 and establish their significance. Determining quantitatively the changes in microearthquake activity is difficult, especially when addressing rate decreases [Wyss and Wiemer, 2000]. Establishing rate decreases within an aftershock sequence is both desirable, because the high seismicity rate allows detailed investigations, and challenging, because establishing the significance of a relative rate change is more complicated when embedded within exponentially decaying sequences rather than in a stationary Poissonian process.

[4] The entire 1997 Kagoshima sequence is not easily fit with existing aftershock sequence models. Ogata [2001] investigated the first 47.8 days of the 1997 Kagoshima aftershock sequence using the earthquake catalog of the Japanese Meteorological Agency (JMA). He found that a two-stage Epidemic-Type Aftershock-Sequence-model (ETAS, [Ogata, 1999; Ogata, 2001]) provides the best approximation although the overall goodness-of-fit throughout the period remains poor. Contrary to the modified Omori law, the ETAS-model considers the magnitude distribution of the aftershock sequence and therefore requires additional free parameters resulting in a limited forecast ability. We apply simpler modified Omori law type models to map the spatial distribution of aftershock sequence parameters because these models allow to compute a more accurate representation of the sequence than just modeling the entire sequence.

\section{Data}

[5] The 1997 earthquake doublet in the Kagoshima Prefecture, Kyushu, southern Japan, occurred on 26 March $\left(\mathrm{M}_{\mathrm{W}}=6.1, \mathrm{M}_{\mathrm{L}}=6.5\right)$ and 13 May $1997\left(\mathrm{M}_{\mathrm{W}}=6.0\right.$, $\mathrm{M}_{\mathrm{L}}=6.3$ ), both followed by rich aftershock sequences. The first mainshock ruptured unilaterally westward for $15 \mathrm{~km}$ along a strike-slip fault striking $\mathrm{N} 280^{\circ}$, while the second mainshock ruptured two orthogonal $7.5 \mathrm{~km}$ long segments, with one being parallel to the first rupture. The hypocenters of the two mainshocks are approximately $5 \mathrm{~km}$ and the parallel rupture planes are approximately $3 \mathrm{~km}$ apart ([Horikawa, 2001], Figure 1).

[6] We analyze the Kagoshima University catalog (KU) [Miyamachi et al., 1999] from 1997.23 to 1997.55 containing about 7000 events in the study area (Figure 1). The KU catalog is preferable to the JMA catalog (about 3500 events), because it has a lower completeness level, relocated aftershock locations, and contains twice as many events. We use the JMA catalog only for cross-validation of our results.

[7] We investigated the magnitude of completeness $M_{\mathrm{c}}$ as a function of time and space [Wiemer and Katsumata, 1999; Wiemer and Wyss, 2000]. The spatial and temporal distribution of $M_{\mathrm{c}}$ is used to establish a lower cut-off magnitude 


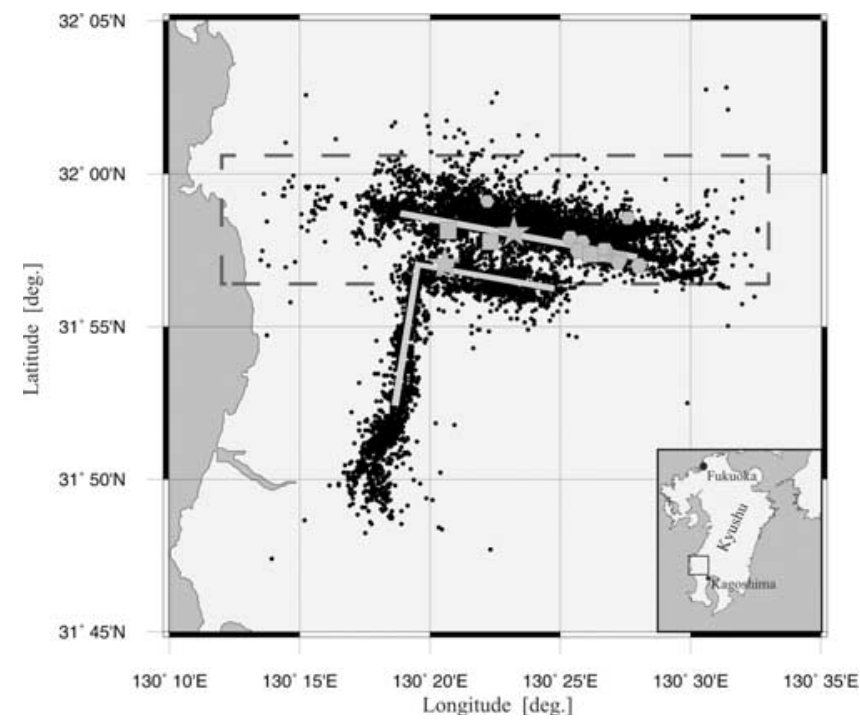

Figure 1. Map of the 1997 Kagoshima earthquake sequence. Fault ruptures are taken from Horikawa [2001], stars indicate the two mainshocks, aftershocks are displayed as squares $\left(5.0 \leq M_{\mathrm{L}} \leq 6.0\right)$ and hexagons $\left(4.0 \leq M_{\mathrm{L}} \leq\right.$ 5.0). As background, the entire seismicity listed in the $\mathrm{KU}$ catalog is plotted as black dots. The dashed gray box frames the area shown in Figure 2.

ensuring that further analysis is not biased by incomplete data. In case of the KU catalog, we set the global minimum threshold at $M_{\mathrm{c}}=1.3$ as results generally fluctuate around $M_{\mathrm{c}}=1.2$ when mapping spatially. In the time series, $M_{\mathrm{c}}$ decreases rapidly from $M_{\mathrm{c}}=1.8$ down to around $M_{\mathrm{c}}=1.2$. The low detection level is partly explained by the installation of temporary stations [Miyamachi et al., 1999]. We also investigated the JMA catalog to determine an overall $M_{\mathrm{c}}$ value in the same manner and obtained a value of $M_{\mathrm{c}}=1.6$.

\section{Method}

[8] To derive the relative rate change values $\Delta_{R C}$, we developed the following procedure: First, we use the observed data of the learning period $t_{L}$ to fit the parameters of four different models of aftershock decay to the aftershock sequence applying the maximum-likelihood method [Ogata, 1999]. Second, the best fitting model is used to forecast aftershocks until the end of the forecast period $t_{\mathrm{F}}$. Finally, relative rate changes are established at the end of the forecast period.

[9] The basic model is the modified Omori law [Utsu, 1961]:

$$
n(t)=k(t+c)^{-p}
$$

with $n(t)$ being the number of earthquakes occurring at time $t$. This is equal to the intensity function $\lambda(t)$ assuming that aftershocks are distributed according to a non-stationary Poisson process. $k$ represents the productivity of the mainshock and depends on the lower magnitude threshold of the earthquake catalog. $p$ is a measure for the exponential decrease of the aftershock rate and $c$ describes a temporal offset that compensates for incomplete data at the beginning of the aftershock sequence. The other three models include one secondary aftershock sequence within the learning period and consist of two superimposed modified Omori laws. We limit our analysis to a maximum of two nested Omori sequences rather than including more complex point process models [Vere-Jones, 1995; Ogata, 1999; Helmstetter and Sornette, 2002] because we believe that simpler modified Omori law models are more suitable for computing forecasts at numerous nodes of a spatial grid. Forecasting the magnitude distribution as required applying an ETAS-model would greatly increase uncertainties.

[10] The intensity function for these nested Omori models is written as:

$$
\begin{gathered}
\lambda(t)=\lambda_{1}(t)=k_{1}\left(t+c_{1}\right)^{-p_{1}} \quad, t \leq t_{a} \\
\lambda(t)=\lambda_{2}(t)=k_{1}\left(t+c_{1}\right)^{-p_{1}}+k_{2}\left(t+c_{2}\right)^{-p_{2}} \quad, t_{a} \leq t \leq t_{L}
\end{gathered}
$$

where $t_{a}$ is the time of the largest aftershock from the mainshock time in the learning period. The indices 1 and 2 relate the modified Omori law parameters in the time periods before and after the occurrence of the largest aftershock at $t_{a}$ in the learning period, respectively.

[11] In summary, we fit four models with increasing model complexity varying as a function of free parameters:

1. Model 1: Modified Omori law (MOL)

(3 parameters: $p, c$ and $k$ )

2. Model 2: nested MOL (4 parameters: $p, c, k_{1}, k_{2}$ )

3. Model 3: nested MOL (5 parameters: $p_{1}, p_{2}, c, k_{1}, k_{2}$ )

4. Model 4: nested MOL

(6 parameters: $p_{1}, p_{2}, c_{1}, c_{2}, k_{1}, k_{2}$ ).

[12] The maximum-likelihood estimate [Ogata, 1999] for each model is computed by a constrained nonlinear grid search over the free parameter space, maximizing the likelihood function. To find the best fitting model we use the corrected Akaike Information Criterion $A I C_{\mathrm{c}}$ [Kenneth et al., 2002]:

$$
A I C_{c}=-2 \max (\ln L)+2(P)+\frac{2 P(P+1)}{N-P-1}
$$

with $\ln L$ being the likelihood function, $P$ the number of free parameters and $N$ the sample size. In contrast to the original AIC, the corrected $A I C_{c}$ penalizes also for the amount of samples which becomes critical for small sample sizes. The $A I C_{c}$ is useful in selecting the best model in the set; however, if all the models are poor, $A I C_{c}$ still selects the one estimated to be best, but even that model may be poor in an absolute sense [Kenneth et al., 2002]. Thus, we estimate the goodness-of-fit for the selected $A I C_{c}$ model to the observed data by applying a Kolmogorov-Smirnov-Test at a significance level of 0.05 [Conover, 1999]. We do not use results for the interpretation of rate changes if even the best model is rejected (rejection level $H$ equal to 1 ).

[13] To establish the significance of a relative rate change, we need an estimate of the uncertainties of the model forecast at time $t_{\mathrm{F}}$, which we derive based on a bootstrap approach [Chernick, 1999]. The original dataset is bootstrapped 100 times, model parameters and forecasts are estimated for each bootstrap sample. From the empirical distribution of forecasted rates, we calculate the $2^{\text {nd }}$ moment as the uncertainty of our forecasts at time $t_{F}$. Thus, we include both, epistemic uncertainties by using different models and aleatory uncertainties due to the data. The 


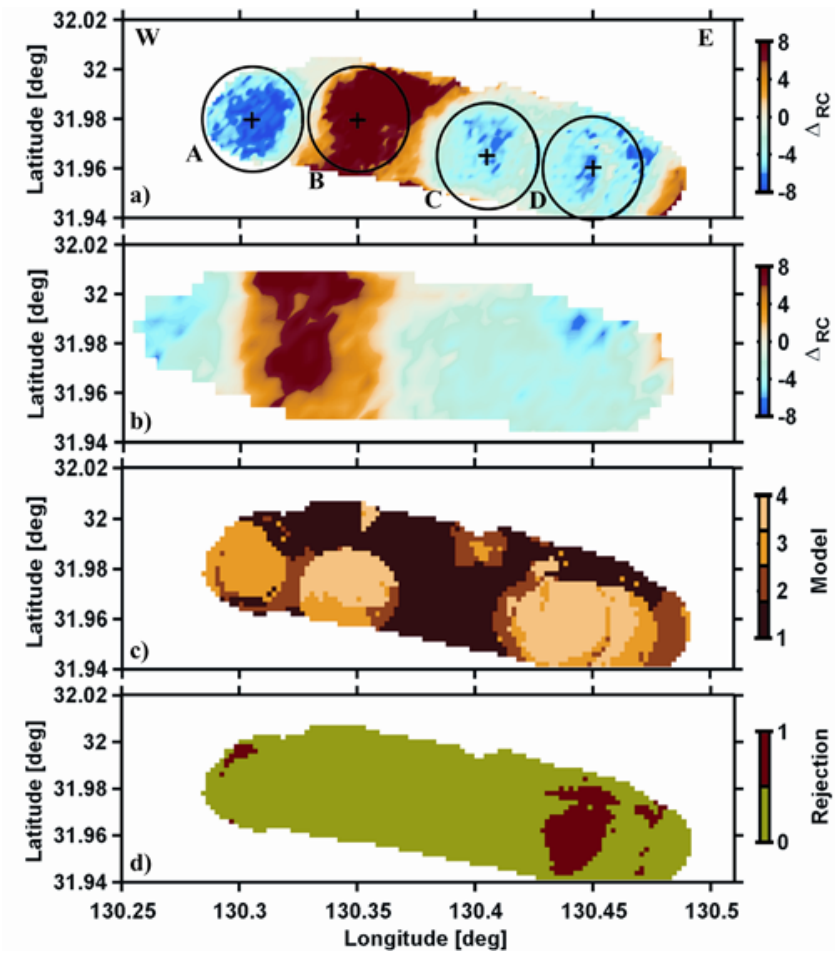

Figure 2. Spatial distribution of a) $\Delta_{R C}$ for the $\mathrm{KU}$ catalog, b) $\Delta_{R C}$ for the JMA catalog. Spatial parameter distributions computed for the KU catalog are, c) the best fitting model and d) the KS-test rejection $(H)$. Sub-catalogs are created using events located within $R=2 \mathrm{~km}$ radii and $R=3 \mathrm{~km}$ for the KU and JMA catalog, respectively. Crosses and circles relate to Figure 3.

relative rate changes are expressed as the ratio of the difference between the cumulative number of observed to forecasted events normalized by the 2 nd moment of the bootstrap forecasts

$$
\Delta_{R C}=\frac{\left(N_{o b s}\left(t_{F}\right)-N_{o b s}\left(t_{L}\right)\right)-\left(\bar{N}\left(t_{F}\right)-\bar{N}\left(t_{L}\right)\right)}{\sigma\left(N_{B s t}\left(t_{F}\right)\right)} .
$$

$N_{\text {obs }}$ is the cumulative number of observed events, $\bar{N}$ the cumulative number of forecasted events at the times $\mathrm{t}_{\mathrm{L}}$ and $\mathrm{t}_{\mathrm{F}}, \sigma\left(N_{B s t}\left(t_{F}\right)\right)$ the 2 nd moment of the bootstrap forecast at the end of the forecast period. The value of $\Delta_{R C}$ expresses the normalized relative rate change between the observed and forecasted events. Positive values report an increase of the observed rate compared to the modeled rate of aftershocks. We map $\Delta_{R C}$ spatially by covering the region with a rectangular grid and sampling events within radius $R$.

\section{Results}

[14] Relative rate changes caused by the second mainshock $\left(\mathrm{M}_{\mathrm{L}}=6.3,13\right.$ May 1997) show strong spatial variations with high statistical significance. The patterns are similar for both, the KU and the JMA catalog analyzed on evenly spaced grids of $0.002^{\circ}$ and $0.005^{\circ}$ degrees, respectively (Figure $2 \mathrm{a}$ and $2 \mathrm{~b}$ ). To evaluate the significance of the rate changes for the KU catalog, we determine the distribution of the best fitting model, and the rejection level
$H$ of the Kolmogorov-Smirnov-test as a measure for the goodness-of-fit (Figures $2 \mathrm{c}$ and 2d). Because the earthquakes exhibited mostly strike-slip faulting with a uniform depth distribution of aftershocks from 0 to $10 \mathrm{~km}$ depth, we chose to show results in map view.

[15] We identify four regions that exhibit significant rate changes (Figure 2a). The detailed temporal development of aftershock activity for four representative grid nodes is shown in Figure 3. The largest relative rate decrease is found at the western end of the aftershock zone of the first mainshock (Figure 2a, circle A). After the second mainshock, the time series of aftershocks at this node (Figure $3 \mathrm{~A}$ ) exhibits a clear rate decrease $\left(\Delta_{R C} \approx-8\right)$. The aftershocks within the adjacent circle $\mathrm{B}$ on the other hand display a large positive $\Delta_{R C}$. This region coincides with the northern end of the north striking-segment of the conjugated fault on which the second mainshock occurred (Figure 1). Hence this positive relative rate change $\left(\Delta_{R C} \approx 11.5\right)$ is most likely triggered aftershock activity near the rupture termination of the second mainshock. Within the third area, to the east of the hypocenter of the first mainshock, we also detected a significant negative rate change (circle $\mathrm{C}$ ). The onset of aftershock quiescence in this volume $\left(\Delta_{R C} \approx-5.5\right)$ occurred about five to seven days later than in circle A. Within the forth area (circle D), close to the east end of the aftershock zone, there is an apparent decrease in aftershock activity following the time of the second mainshock. However, the model fit in the learning period is poor (Figure 3D) as indicated by the KS-test rejection level $H=1$, thus we do not consider this region to be meaningful. At the south eastern end, we find significant positive rate changes for off-fault aftershocks but insignificant ones closer to and north of the fault line.
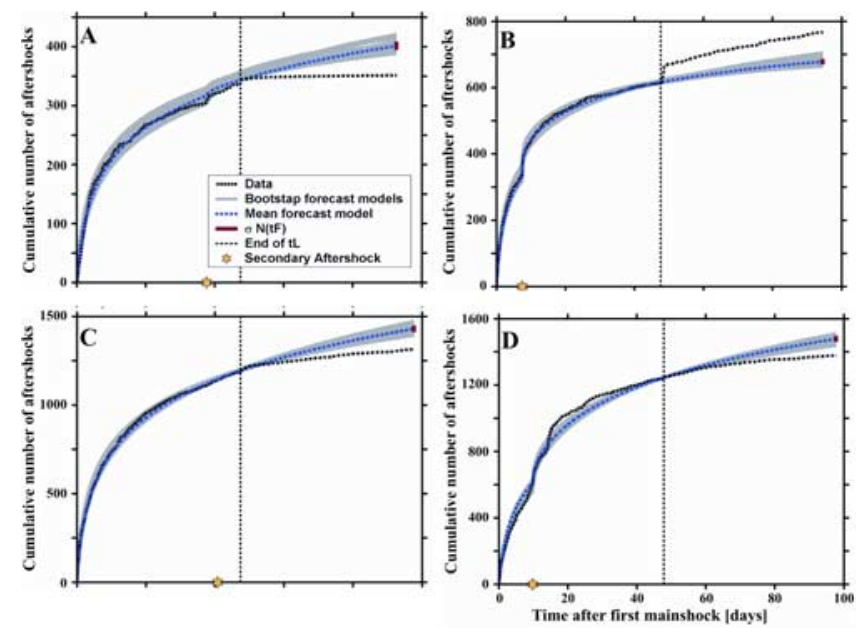

Figure 3. Modified Omori law fits for grid nodes indicated on Figure $3 \mathrm{a}$ with the bootstrap forecast models plotted as gray lines in the background, the dashed blue line displaying the mean forecast model and the red bar indicating the standard deviation of forecasted events at time $t_{\mathrm{F}}$. The vertical line indicates the end of the learning period $t_{\mathrm{L}}$. All panels show significant rate changes with the ones in panel $\mathrm{A}-\mathrm{C}$ not rejected by the KS-test $(H=0)$. In contrast, panel D shows a poor fit as even the best model is rejected by the $\mathrm{KS}$-test $(H=1)$. 
[16] The nested Omori models that match the aftershock data vary spatially (Figure 2c) because the large aftershocks have a heterogeneous spatial distribution (Figure 1). The regions $\mathrm{A}, \mathrm{B}$, and $\mathrm{D}$ of rapidly changing aftershock activity require the nested Omori models 3 or 4 due to the complexity of the aftershock occurrence: Region A is characterized by a series of $3.0 \leq M_{\mathrm{L}} \leq 3.9$ events, the sequence in region $\mathrm{B}$ is influenced by a strong $M_{\mathrm{L}}=5.6$ event and region $\mathrm{D}$ is again characterized by a series of $4.0 \leq M_{\mathrm{L}} \leq$ 4.8 events. In contrast, region $\mathrm{C}$ does not include large aftershocks and is well fitted with a single modified Omori law. Thus, the respective spatial pattern of the model distribution concerning their complexity coincides well with the aftershock distribution.

\section{Discussion and Conclusions}

[17] To quantify the effects of the second mainshock on the aftershock sequence of the first mainshock, we investigated the aftershock activity focusing on aftershocks adjacent to the northern fault that ruptured in the first mainshock. The 1997 Kagoshima earthquake doublet occurred in a small area, and thus it is reasonable to expect the second mainshock to have influenced the ongoing aftershock sequence of the first. We applied an objective spatio-temporal mapping approach and formulated a measure of the statistical significance of rate changes within the decaying aftershock sequence. Thus our approach differs from Stein [2003] and Toda and Stein [2003] who focused mostly on the physical implications of off-fault earthquake triggering and used subjectively selected boxes to determine rate changes. Our high resolution mapping results confirm quantitatively only some of their qualitative results. In particular, our mapping shows that a statistically significant rate decrease followed the second Kagoshima mainshock in the westernmost part of the aftershock zone of the first mainshock (Figure 2a and 3A, box A in [Toda and Stein, 2003, (Figure 6)]). We also identify two other on-fault regions of increased and decreased aftershock activity. However, at the eastern end of the aftershock zone, we find statistically insignificant rate variations if possible to detect due to sparse data, where Toda and Stein, [2003] claimed a qualitative significant rate decrease (Figure $3 \mathrm{a}$, box $\mathrm{C}$ in [Toda and Stein, 2003, (Figure 6)]). Our quantitative analysis shows that both off-fault and on-fault aftershock activity were increased but also decreased by the nearby second mainshock. These findings may independently contribute to determining the validity of the stress triggering hypothesis [Harris, 1998] as well as seismicity rate change computations based on rate and state friction [Dieterich, 1994] by using our approach as a quantitative measure.

[18] How the effects of static stress changes on earthquake triggering may be affected by the presence of crustal fluids or anisotropic crustal material properties is poorly understood. There are many hot springs in the area and crustal fluids or geothermal areas as well as geological boundaries may have affected the temporal evolution and spatial distribution of aftershocks [Miyamachi et al., 1999]. Furthermore, differences in material properties could have contributed to the rupture behavior of the two mainshocks and the spatiotemporal distribution of the aftershocks. The east - west rupture of the second mainshock propagated eastward while the rupture of the first mainshock propagated to the west [Horikawa, 2001]. Miyamachi et al. [1999] relocated the 1997 Kagoshima earthquakes using a 3-D velocity model and suggested that some of the aftershocks were occurring on geological boundaries striking north - south. Thus, several other geophysical and geological parameters as well as other mechanisms, e.g., coseismic release of trapped highpressure fluids, may have had similar influence on the spatial and temporal development of this sequence than coseismic changes in static stress, but these cannot be differentiated with our approach.

[19] Acknowledgments. We thank H. Miyamachi, K. Goto and S. Toda for providing us with the Kagoshima University catalog, A. Yoshida for the JMA catalog. We thank M. Mai, D. Schorlemmer, S. Miller, G. Zöller and one anonymous reviewer for comments that improved the manuscript significantly. This is contribution number 1332 of the Institute of Geophysics, ETH Zurich. E. Hauksson was supported by USGS grant number 01HQGR038 and this is contribution number 9034 of the Division of Geological and Planetary Sciences, California Institute of Technology, Pasadena.

\section{References}

Chernick, M. R. (1999), Bootstrap methods: A practitioner's guide, John Wiley, New York.

Conover, W. J. (1999), Practical Nonparametric Statistics, John Wiley, New York.

Dieterich, J. (1994), A constitutive law for rate of earthquake production and its application to earthquake clustering, J. Geophys. Res., 99(B2), $2601-2618$.

Harris, R. A. (1998), Introduction to special section: Stress triggers, stress shadows, and implications for seismic hazard, J. Geophys. Res., 103(B10), 24,347-24,358.

Helmstetter, A., and D. Sornette (2002), Subcritical and supercritical regimes in epidemic models of earthquake aftershocks, J. Geophys. Res., 107(B10), 2237, doi:10.1029/2001JB001580.

Horikawa, H. (2001), Earthquake doublet in Kagoshima, Japan: Rupture of asperities in a stress shadow, Bull. Seismol. Soc. Am., 91(1), 112-127.

Kenneth, P., K. P. Burnham, and D. R. Anderson (2002), Model selection and multimodel inference: A practical information - Theoretic Approach, Springer, New York.

King, G. C. P., R. S. Stein, and J. Lin (1994), Static Stress Changes and the Triggering of Earthquakes, Bull. Seismol. Soc. Am., 84(3), 935-953.

Miyamachi, H., K. Iwakiri, H. Yakiwara, K. Goto, and T. Kakuta (1999), Fine structure of aftershock distribution of the 1997 Northwestern Kagoshima Earthquakes with a three-dimensional velocity model, Earth Planets and Space, 51(4), 233-246.

Ogata, Y. (1999), Seismicity analysis through point-process modeling: A review, Pageoph., 155(2-4), 471-507.

Ogata, Y. (2001), Increased probability of large earthquakes near aftershock regions with relative quiescence, J. Geophys. Res., 106(B5), 8729-8744.

Stein, R. S. (2003), Earthquake conversations, Sci. Am., 288(1), 72-79.

Toda, S., and R. S. Stein (2003), Toggling of seismicity by the 1997 Kagoshima earthquake couplet: A demonstration of time dependent stress transfer, J. Geophys. Res., 108(B12), 2567, doi:10.1029/2003JB002527.

Utsu, T. (1961), A statistical study on the occurrence of aftershocks, Geophys. Mag., 30, 521-605.

Vere-Jones, D. (1995), Forecasting earthquakes and earthquake risk, Int. J. Forecasting, 11(4), 503-538.

Wiemer, S., and K. Katsumata (1999), Spatial variability of seismicity parameters in aftershock zones, J. Geophys. Res., 104(B6), 13,13513,151 .

Wiemer, S., and M. Wyss (2000), Minimum magnitude of completeness in earthquake catalogs: Examples from Alaska, the Western United States, and Japan, Bull. Seismol. Soc. Am., (90), 859-869.

Wiemer, S., and M. Wyss (2002), Mapping spatial variability of the frequency-magnitude distribution of earthquakes, Adv. Geophys., 45, $259-302$.

Wyss, M., and S. Wiemer (2000), Change in the probability for earthquakes in Southern California due to the Landers magnitude 7.3 earthquake, Science, 290, 1334-1338.

E. Hauksson, California Institute of Technology, Los Angeles, CA, USA. S. Neukomm, S. Wiemer, and J. Woessner, Institute of Geophysics, ETH Zurich, Switzerland. (woessner@seismo.ifg.ethz.ch) 